\title{
Physical Bases of Medical Ultrasound
}

\section{Jasminka Brnjas-Kraljevic}

Department of Physics and Biophysics, School of Medicine, University of Zagreb, Croatia

Correspondence: Jasminka Brnjas-Kraljevic, Department of Physics and Biophysics, School of Medicine, University of Zagreb 10000 Zagreb, Croatia, e-mail: kraljevi@mef.hr

\section{INTRODUCTION}

The ultrasound diagnostic has proven itself as a rich and informationfull method in obtaining structural and functional images of our body. We can learn about the function of our body and recognize its malfunction. The bottom idea behind all medical diagnostic methods is first to perturb the system by applying a defined amount of energy and than to collect the information about the system by measuring either the perturbations coursed by the interaction or the processes of relaxation. To understand any of the method and to be able to extract the information content in the images physical principles have to be understood. Therefore, we would learn about the nature of the ultrasound and go through some physical principles of the ultrasound interaction with our tissue and how that reflects on images.

\section{THE SOUND WAVE}

The energy applied in the ultrasound diagnostic is mechanical energy of the sound wave. The wave is physical appearance by which the energy is propagating through space. In the sound wave the energy propagating is the mechanical energy of particle oscillations. Mechanical means that it courses oscillations of the particles in the propagating medium. That can result in tissue damages, and it surely is the course of heating ability of propagating sound wave. At the same time that means that the sound wave can not propagate through space without particles. The sound wave is described by parameters: ${ }^{1}$ wave length $(\lambda)$, frequency $(f)$, time period $(T)$, amplitude $(A)$ and velocity of propagation $(v)$. Parameters are connected by simple relationships $\lambda=v T, f=1 / T$.

The source of the sound wave is body that oscillates in an elastic medium and the frequency of wave is the frequency of source oscillation. The sound wave propagates mainly as a longitudinal wave. The particles carrying the mechanical energy through the space are oscillating in the direction of the wave

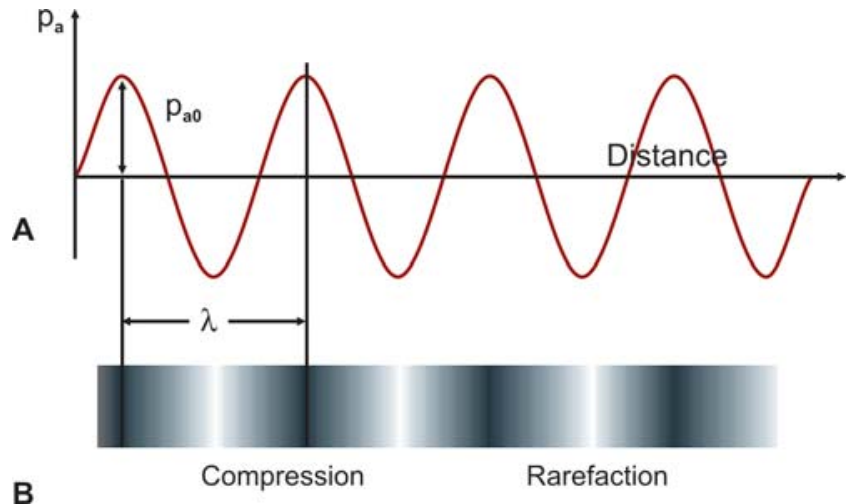

Figs 1A and B: The sound wave: $(A)$ distribution of audible pressure in the medium of sound propagation in dependence on distance from source in the moment of observation; $(B)$ distribution of compression and rarefaction of particles because their oscillations in the direction of wave propagation

propagation. The result of such propagation is regular changes of local pressure and density in the medium of propagation (Figs $1 \mathrm{~A}$ and $\mathrm{B}$ ).

The acoustic velocity of wave propagation is determined by elastic properties and the density of the medium. It is a sensitive function of temperature while its dependence on frequency is minimal in the frequency range of diagnostic ultrasound. The slowest propagation is in the air, only $344 \mathrm{~m} / \mathrm{s}$, in homogeneous liquids increases with increasing viscosity and in solid, elastic medium is mainly determined by Young's elastic modulus. In inhomogeneous medium, as is our body, the dependence of velocity on density and viscosity could be very complicated function. The parameter to describe tissue diversity in sound wave propagation ability is acoustic impedance of the tissue. It is defined as the product of acoustic velocity in the medium and the density of the medium, $\boldsymbol{Z}=\boldsymbol{\rho} \boldsymbol{v}$. Data for some biological tissue are listed in Table 1.

The intensity of the sound wave is the measure of energy that sounds wave caries through the space. It is the velocity of

\footnotetext{
${ }^{1}$ Frequency is the number of perturbations in one second. The unit is $\mathrm{Hz} ; 1 \mathrm{~Hz}$ equals 1 oscillation in one second. Period is the time of one oscillation measured in seconds. Amplitude is maximal displacement of the oscillating particle from the equilibrium position. Wave length is the distance between two adjacent amplitudes.
} 
Table 1: Acoustic parameters for biological tissues at room temperature

$\begin{array}{lrrrr} & \begin{array}{r}\text { Density } \\ \mathrm{kgm}^{-3}\end{array} & \begin{array}{r}\text { Velocity } \\ \mathrm{ms}^{-1}\end{array} & \begin{array}{r}\text { Impedance } \\ 10^{-6} \mathrm{~kg} / \mathrm{m}^{2} \mathrm{~s}\end{array} & \begin{array}{r}\alpha \\ \mathrm{dB} / \mathrm{cm}\end{array} \\ \text { Air } & 1.20 & 344 & 0.00043 & 11.9 \\ \text { Lung } & 400 & 650 & 0.26 & \\ \text { Fat } & 920 & 1467 & 1.33 & 0.60 \\ \text { Water } & 1000 & 1520 & 1.48 & 2.2 \times 10^{-4} \\ \text { Brain } & 1030 & 1504-1612 & 1.55-1.66 & 0.85 \\ \text { Kidney } & 1040 & 1558 & 1.62 & 0.78 \\ \text { Liver } & 1566 & 1566 & 1.66 & 0.96 \\ \text { Myocardium } & 1070 & 1561-1626 & 1.67-1.74 & 1.3-3.2 \\ \text { Skull bone } & 1380-1810 & 2717-4077 & 3.75-7.38 & 11.3-20\end{array}$

the energy $E / t$ carried through the area of $1 \mathrm{~m}^{2}$ perpendicular to the propagation direction. Intensity, $I=E / t S$, is measured in $\mathrm{W} / \mathrm{m}^{2}$. Intensity of the sound wave is important parameter in medicine diagnostic. It measures energy of interaction of sound wave and biological tissue, meaning that it determines the possible consequences of the applied diagnostic procedure. Intensity can also be expressed by parameters connected with properties of sound wave source and medium acoustic properties: $2 I=A^{2} \omega^{2} \rho v$, where $\mathrm{A}$ is the amplitude and $\omega$ is the frequency of the sound source and $\rho v$ is the acoustic impedance of the medium. In the expression for intensity amplitudes and frequencies are in inverse relationship meaning that for defined intensity the amplitudes for higher frequencies waves will be smaller. We have already mentioned that the sound wave could be presented as regular interchange of regions of compression and rarefaction and as the consequence the regular change of local pressure. The pressure difference when there is a sound wave propagating and when there is no wave is acoustic pressure. The intensity can be also presented as a function of pressure amplitude of the harmonic acoustic wave: $I=p_{a 0}{ }^{2} /(2 \rho v)$.

Depending on how our body reacts to the mechanical energy of the sound waves we recognize three main frequency domains: infrasound, audible sound and ultrasound. Audible sound is in the frequencies interval of $20 \mathrm{~Hz}$ to $20 \mathrm{kHz}$ and the intensity interval from $10^{-12}$ to $10 \mathrm{~W} / \mathrm{m}^{2}$.

Infrasound is in domain of frequencies lower than $20 \mathrm{~Hz}$. Those are the frequencies of sound waves of earthquake and change of atmospheric pressure. Our inner organs have resonant frequencies in the domain of infrasound frequencies.
Ultrasound is in the domain of frequencies higher than 20 $\mathrm{kHz}$. The interactions of these frequencies waves and our body are negligible and therefore these energies can be used for diagnostic procedure. For medical diagnostic the frequencies of 1-20 MHz are used and intensities needed for diagnostic are 10 to $10^{3} \mathrm{~W} / \mathrm{m}^{2}$ (in medical textbooks usually expressed as $\left.1-100 \mathrm{~mW} / \mathrm{cm}^{2}\right)$.

\section{PIEZOELECTRIC CRYSTAL IS THE SOURCE AND THE DETECTOR OF ULTRASOUND}

The source of ultrasound waves is a piezoelectric crystal. These materials have the ability to transform the energy of oscillating electric field into mechanical energy of sound waves by the process named inverse piezoelectric effect. ${ }^{2}$ Also when exposed to the mechanical perturbation of sound wave the crystal transforms the mechanical energy of deformation into voltage across the side surfaces by process piezoelectric effect, and offers the measurement of sound wave parameters. Placed in the elastic medium the crystal can be both, the source and the detector of the ultrasound waves. The efficiency of energy transformation in both directions, electrical into mechanical when source or mechanical into electrical when detector, is extremely sensitive upon crystal thickness. The resonant conditions, the best energy transformation, are achieved when the crystal own frequency is identical to the frequency of the sound wave. It is realized for thickens of $\lambda / 2$. The intensity of the sound wave, on the other hand, is independent of crystal geometry. Hence, the same electromechanical transductor can act as the source of the wave with high intensity, and the detector for very low intensity signals. Mainly, ultrasound probe (US-probe) is produced of very thin crystal, few tenths of mm. The most used materials are ferroelectric ceramic, lead zirconat titanate (PZT) or plastic, polyvinylidene difluoride (PVDF). These materials are highly piezoelectric and have advantage that can easily be shaped differently. The crystal is sandwiched by film-thin silver electrodes on opposite sides of the crystal. The electrode towards the body is always grounded. When the electrode opposite is by coaxial cable connected to voltage source the transducer is the US-source, and when connected to oscilloscope it is US-detector. To achieve good resolution ultrasound is emitted in short pulses duration of $1 \mu$ s or shorter. Therefore the crystal oscillations must be quickly attenuated. It is easier

\footnotetext{
${ }^{2}$ Brothers Jacques and Pier Curie have described and explained the piezoelectric effect for the first time in year 1880 . They noticed that mechanical deformations of some crystal induced voltage across its opposite surfaces. When placed in the alternating electric field crystal undergoes mechanical deformation. Induced oscillations of the crystal had the frequency of the alternating electric field. Very important observation was that the response of the crystal to the outer stimulus was extremely fast and so very high frequency oscillations could be induced; from $10^{5}$ to $10^{9} \mathrm{~Hz}$.
} 
Table 2: The parameters for commercial ultrasound apparatuses

$\begin{array}{ll}\text { Frequency } & 0.5-20 \mathrm{MHz} \\ \text { Pulse length } & 0.2-2 \mathrm{~ms}^{-1} \\ \text { Pulse repetition rate } & 50-1500 \mathrm{~s}^{-1} \\ \text { Mean acoustic strength } & 0.25-25 \mathrm{~mW} \\ \text { Intensity } & 1-100 \mathrm{~mW} / \mathrm{cm}^{2}\end{array}$

to achieve with high frequencies. The higher frequencies transducer offers better resolution power. But unfortunately the usage of higher frequencies is limited to the structures near the source because of mighty attenuation of high frequencies in the tissues. To achieve qualitative images the emitted sound wave bundle has to be narrow and directed only in determined direction. Therefore, there is a massive attenuator in the background of the crystal and the whole probe is wrapped up in acoustic insulator (Fig. 2).

In Table 2 the usual values for diagnostic apparatuses are listed.

The diagnostic method is very simple. The US-probe usually used on the surface of the body sends short pulses of high frequencies into the body. Time needed for the reflected pulse to return to the source is the information about the position of different structures on the path of pulse propagation. But because absorption of sound energy in the body increases with frequency the resolution is poorer for deeper tissues. Therefore some new probes are developed that can be introduced into the body cavities, transvaginal probe, transrectal probe or transesophageal probe, and can use higher frequencies and achieve better imaging of inner organs.

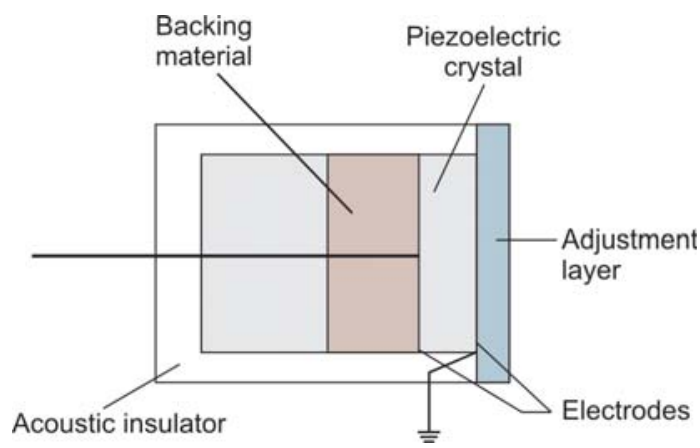

Fig. 2: The ultrasonic probe: The thickness of crystal is $\lambda / 2$ to meet resonance conditions; the adjustment layer is $\lambda / 4$ thick and has adjusted impedance to achieve maximal energy transfer into the tissue; sound insulator blocks the sound propagation in all directions so that directed plane US-wave is emitted
The different shapes of transducers are used for different purposes. The linear probe produces rectangular image and is used for surface tissues. Sector probe gives triangle image and is used for heart observation and sometimes for inner organs. Convex probe is used for inner organs.

\section{ATTENUATION OF THE SOUND WAVE}

When the ultrasonic wave is propagating through a heterogeneous medium its energy decreases as a function of distance. The energy is reduced because it is absorbed or reflected or scattered by the medium. Reflected and scattered energy has just changed the direction of propagation, but the absorbed sound energy due to frictional effects mainly converts into heat.

Energy absorption is detected as the reduction of sound wave amplitude and consequently sound wave intensity or audible pressure. The decrease in intensity is exponential function of depth, $x: I(x)=I(0) e^{-\alpha x}$ or , $p(x)=p(0) e^{-\alpha^{\prime} x}$ where, $\alpha$ is the absorption coefficient ${ }^{3}$ characteristic for the tissue and dependent on wave frequency. It is bigger for higher frequencies, meaning that the higher frequencies are better absorbed in the tissues. The half-value thickness is the tissue thickness needed to decrease the starting intensity to its half value, $X_{12}=\frac{\ln n_{2}}{2 \alpha}$. The half-value thickness decreases with increasing frequencies, and that put the limit to the maximum frequency that can be clinically used.

There is small benefit of the absorption for diagnostic processes, mainly because of the complicated functional dependence of attenuation on frequency. Sometimes it is used for imaging symmetric organs and to compare their functions.

\section{REFLECTION AND TRANSMISSION}

When the wave meets an interface between two media part of the energy will be transmitted and part of it reflected backward. The two media are characterized by acoustic impedances. Reflected wave holds its velocity but changes the direction. The transmitted wave continues to move forward with slightly changed direction but at different velocity. If the wavelength is much smaller than the dimension of the interface the classical wave laws of reflection and refraction can be applied (Fig. 3). For reflected waves reflected angle is equal to incident one:

\footnotetext{
${ }^{3}$ The absorption coefficient is measured in decibels/cm or in neper/cm. When measured in $\mathrm{dB}$ it is defined by intensity ratio $\alpha=10 \ln \frac{I(x)}{I(0)} n p / c m$ and in nepers by the ratio of acoustic pressure $\alpha^{\prime}=\frac{1}{x} \ln \frac{p(0)}{p(x)} n p / c m$. The conversion is $\alpha(d B / c m)=8.686 \alpha$ (np/cm)
} 


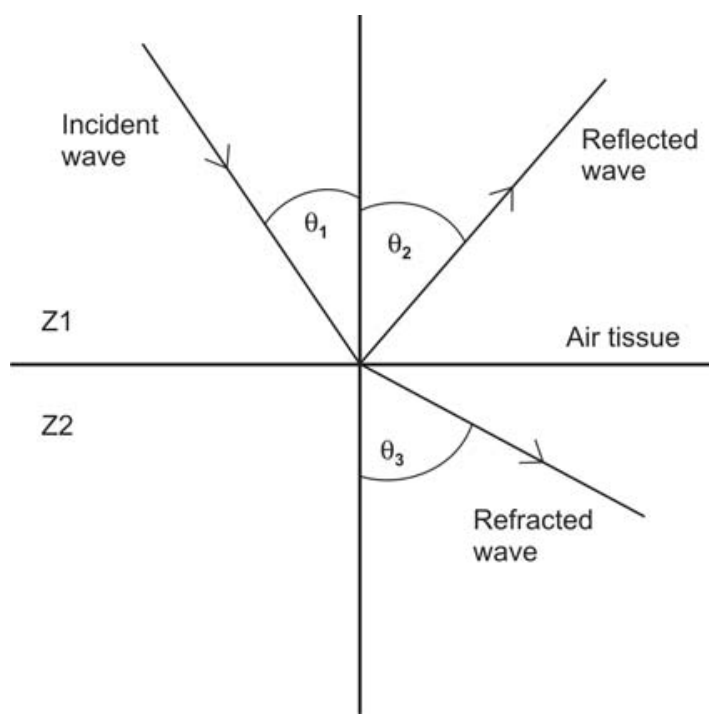

Fig. 3: Reflection and refraction of plane wave on flat boundary: The ratio of acoustic impedances determines the intensities of reflected and of transmitted waves. The angles of reflection and refraction are determined only by the velocity of sound propagation in the media

$\boldsymbol{\theta}$ (reflected) $=\boldsymbol{\theta}$ (incident), and for transmitted wave the angle depends on the velocity of propagation in the two media: $\frac{\sin \theta_{i}}{v_{i}}=\frac{\sin \theta_{t}}{v_{t}}$. If the incidence angel is zero the transmitted wave does not change the direction and the reflection is backward. This fact is very important as it is used by diagnostic procedure.

The transmitted and reflected energies of the sound waves are entirely dependent on the acoustic impedances of the two media building the boundary. The pressure/intensity reflection and transmission coefficients are calculated obeying the boundary conditions: the pressure and particle velocity should be continuous across the boundary:

$$
\frac{I_{v}}{I_{0}}=\left(\frac{Z_{2} \cos \theta_{1}-Z_{1} \cos \theta_{3}}{Z_{2} \cos \theta_{1}+Z_{1} \cos \theta_{3}}\right)^{2} \text { and } \frac{I_{2}}{I_{0}}=\left(\frac{4 Z_{2} Z_{1} \cos ^{2} \theta_{1}}{Z_{2} \cos \theta_{1}+Z \cos \theta_{3}}\right)^{2}
$$

If the incidence is normal to the boundary (incidence angle is 0 ) then the intensity coefficients are:

$$
\frac{I_{v}}{I_{0}}=\left(\frac{Z_{2}-Z_{1}}{Z_{2}+Z_{1}}\right)^{2} \text { and } \frac{I_{2}}{I_{0}}=\left(\frac{4 Z_{2} Z_{1}}{Z_{2}+Z}\right)^{2}
$$

It is important to stress out that at the interface of two media with approximately the same acoustic impedances the transmission is maximal, and the reflection is neglectable. The maximal reflection is on the border of two media with very different acoustic impedances. For instance on the border of two soft tissues: muscle and fat, only $1 \%$ of acoustic energy is reflected. It is just enough to detect this border. On the border of soft tissue and bones, reflection is very strong. The intensity transmitted through bone is very small or none and borders behind it can not be detected. On the border of air and soft tissue the reflection is nearly complete (99.9\%). This mismatch of impedances is overcome with the special gel used on the body surface to eliminate the air bubbles between transducer and the body. This mismatch is also the main reason why the inner volume of the lung can not be examined by the ultrasound.

In diagnostic method the interfaces of very different impedances as well as one with similar ones must be detected. For the transmission from generator into the body the echo signal should be very small, most of the energy should be transmitted into the body, so the impedances should be similar. On the other hand for successful imaging with the ultrasound even the interfaces with small differences in impedances must reflect measurable signals.

The basic image of the biological tissue is produced by sound waves reflected from the interfaces perpendicular to the direction of propagation. Added information is obtained by the scattering on small inhomogeneities. The scattering and reflection are dependent on the elastic properties of the tissues, so one my postulate that echographic visualization of the tissue is determined mostly by their connective tissue content. The connective tissue and the tissue containing high concentration of connective tissue have high acoustic impedances. The $Z$ of blood vessels is higher than for surrounding tissues and therefore are more visualizable than the other soft tissues. But it has to be stressed that the attenuation of the sound in the connective tissue is also very high. The half with is very small (for collagen $2.7 \mathrm{~cm}$ for $1 \mathrm{MHz}$ ) so that very little intensity would transmit through such tissue. The structures behind would not be visible. We are talking about acoustic shadow.

\section{DOPPLER EFFECT}

Doppler effect is the phenomenon in which the detected frequency is not identical to the frequency of the source. (We have mentioned that the frequency is determined by the frequency of the oscillating source and does not change in different media). The Doppler effect can be observed when the detector or the source is relatively moving Figures $4 \mathrm{~A}$ and $\mathrm{B}$. Because the velocity of object movement is added to the velocity of sound propagation the measured change in frequency will depend on the direction of relative movement of the objects. If one of both, the source or the detector, is still the frequency detected will increase for approaching and will decrease for departing. If both are moving the new sound frequency is determined by $f_{p}=f_{0} \frac{v+v_{d}}{v-v_{s}}$ where $f_{0}$ is the emitted frequency 

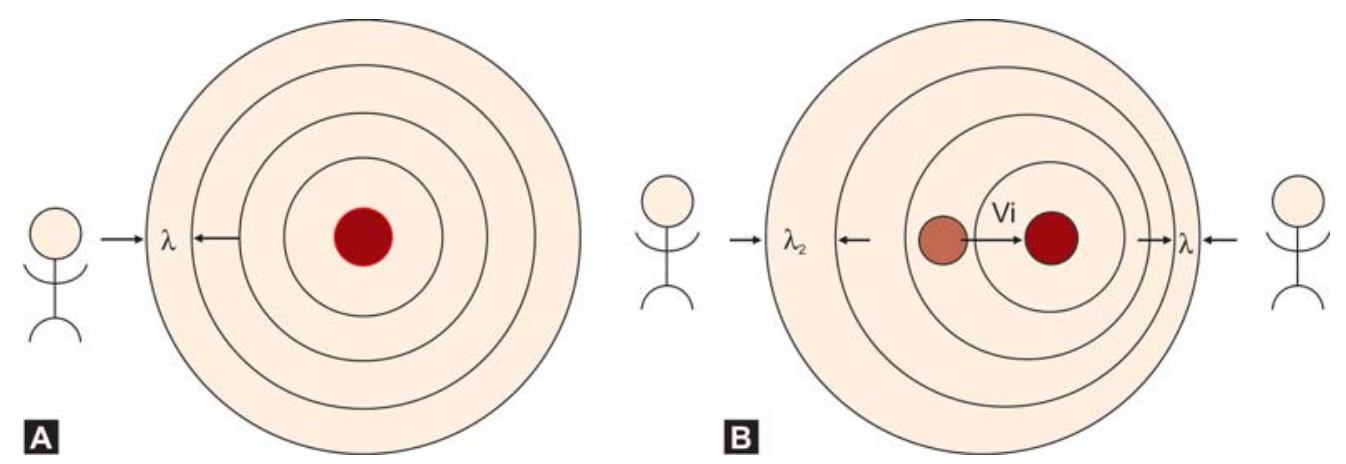

Figs 4A and B: The Doppler effect: (A) If both source and detector are stationary the detected frequency is identical with emitted one. (B) If the source is approaching stationary detector will detect higher frequency, and if drifting apart, measured frequency will be lower. The difference of the measured frequency and actual source frequency is Doppler shift. It depends on the velocity of the moving object, source or detector or both

and $f_{p}$ is detected frequency and $v, v_{d}, v_{s}$ are velocities of the sound, the detector and the source respectively. The difference of the frequencies $\Delta f=f_{0}-f_{d}$ named Doppler shift is:

$$
\Delta f_{d}=f_{0}-f_{0} \frac{v+v_{d}}{v-v_{s}}=f_{0} \frac{v_{s}-v_{d}}{v}
$$

In diagnostic measurements the echo beam is detected by still detector, so the Doppler shift can be measured only if the reflecting border is moving. This border is moving detector and at the same time moving emitter of the sound. The detector measures frequency shift in dependence on velocity of reflecting border, $v_{b}$

$$
\Delta f=f_{0} \frac{2 v_{b}}{v}
$$

\section{ECHOSONOGRAPHY}

Reflection and scattering of the ultrasound wave in heterogeneous biological tissues are used in two diagnostic methods. Echosonography, based on reflection, provides information abut size and geometry of the organs and the method of coherent scattering adding the detailed information about regular structure within the organs.

The advantage of echosonography in diagnostic is unquestionable. The time needed for the emitted US-pulse ${ }^{4}$ to reach the reflecting interface in the tissue and to return back to the source is measured. All the returned pulses are detected as function of time of delay. The intensity of returned pulse reflects the ratio of acoustic impedances, but it is strongly dependent on the absorption along the path of propagation. Hence, the diagnostic value is in measured time intervals between reflected pulses and not in intensities of the echoes. The image is the distribution of reflecting interfaces from the body surface to the spine, but only the ones which are perpendicular to the propagation direction.

\section{RESOLUTION OF THE ECHO METHOD}

The shape of the US-pulse determines the resolution abilities of the method. Dimensions and properties of US-pulse are changing with the penetration into the body and that primarily determines the quality of the image. If we observe the ultrasound beam in the whole system we can define two different zones. The first one strait to the emitter is Fresnel zone. The shape and dimensions of it are determined by the shape and dimensions of the emitter and by the frequencies of the wave. The intensity is oscillating with wave frequency. And the second is Fraunhofer zone, apart from emitter, in which the bundle is diverging and the intensity is uniformly decreasing with distance. Resolution is better preserved into depth if the Fresnel zone is longer (Fig. 5).

The audible field in the vertical section of the sound bundle is nonhomogeneous, but has distribution of intensities in the plain perpendicular to the direction of propagation. In medicine is routinely measured the SP-spatial peak intensity and SAspatial average intensity. These two parameters are important

\footnotetext{
${ }^{4} \mathrm{Pulse}$ is the time limited emission of sound waves what defines very precisely the amount of mechanical energy delivered to the body. By standard the pulse lasts for 2-3 wavelengths. It can be calculated by multiplying I and number of oscillations and expressed in $\mathrm{cm}$. The pulse duration measured in seconds is the ratio of oscillation number and wave frequency. For higher frequencies the pulses are shorter. The resolution decreases with penetration in the tissue. It is explained by common physical phenomena characteristic for sound wave movements. The source emits defined frequencies interval around the nominal one. While penetrating through the body the higher frequencies are strongly absorbed what lengthened space duration of the pulse and courses worse resolution.
} 

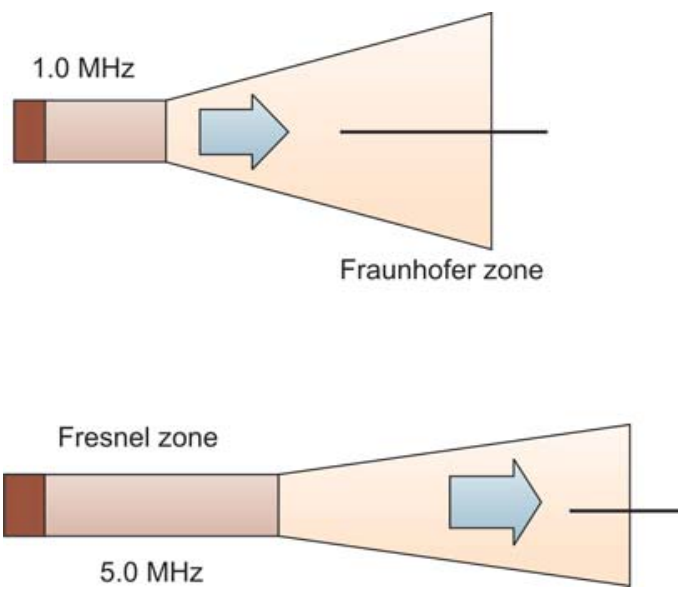

Fig. 5: The characteristics of US-bundle: The shape of near, Fresnel zone depends on the dimension (D), of the transducer and wave length $(\lambda)$ of the sound wave. In the length of that zone the intensity is changing rapidly but the width of the beam remains fixed. Beyond that distance, $D^{2} / 4 \lambda$, is far zone or Fraunhofer zone. The intensity decreases smoothly and the width of the beam increases with the distance

by determination of biological effects of ultrasound at the biological system (Fig. 6).

Quality of the ultrasound image is defined by the resolution which can be achieved. Resolution is the smallest distance between two details in the tissue that can be separated on the image. Resolution is measured in mm. Maximal resolution is limited by wave length and pulse width. We are talking about two resolutions; one in the direction of ultrasound propagation, axial resolution and lateral resolution, perpendicular to the propagating direction (Figs 7A and B).

Axial or longitudinal resolution is the minimal measurable distance between two reflecting interfaces on the propagating path. It is limited with spatial pulse length, SPL (multiplication of the number of oscillations in one pulse with wavelength of the sound). Time between two adjacent echoes can be measured only if pulse SPL is at least two waves length. Hence, maximal resolution is determined by the wavelength. Longitudinal relaxation, SPL/2, is better for higher frequencies applied in diagnostic. The whole time we must bear in mind that resolution is decreasing with penetration into the tissue, because of higher absorption for waves with higher frequencies. ${ }^{5}$

To visualize the structure of reflecting surface it is necessary to resolve the reflection from two parallel interfaces

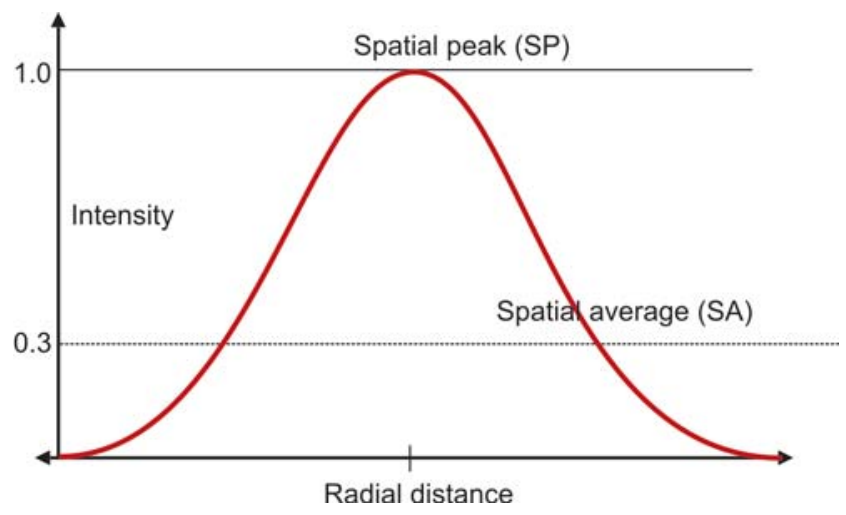

Fig. 6: Lateral bundle profile in far zone: A transverse intensity profile of the sound bundle primarily determines lateral resolution. The width of the bundle is defined as the width at spatial average intensity. Two objects can be resolved if they are apart more than the width of the bundle

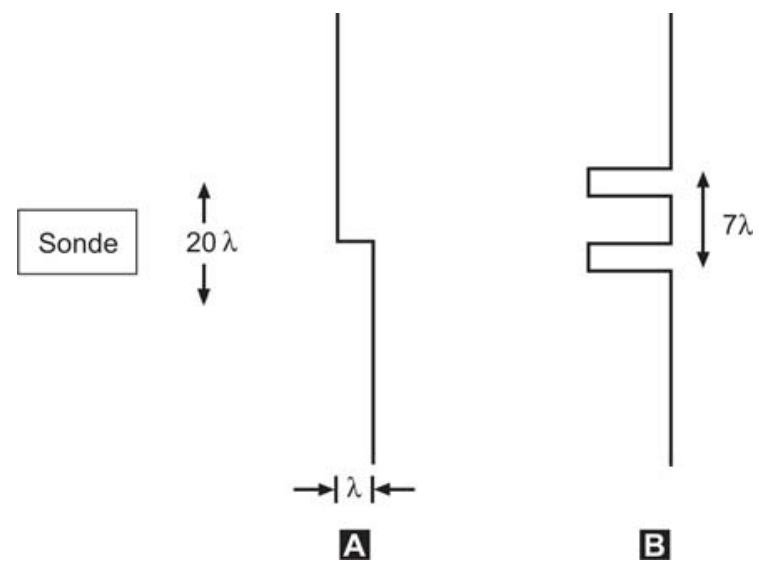

Figs 7A and B: Axial and lateral resolution: (A) Axial resolution is along the direction of propagation. It depends ultimately on the wavelength. The pulses must be short so that two echoes from nearby boundaries do not overlap. The length of the pulse should be $1 \mu \mathrm{s}$, what is for $10 \mathrm{MHz}$ sound only about ten oscillations. (B) Lateral resolution is less because it depends on pulse width which is determined by the dimension of the transducer

perpendicular on the direction of wave propagation. That is lateral resolution. Lateral resolution is always less than longitudinal resolution. It is in the range of several wavelengths and depends on the geometry of the ultrasonic probe, frequency of the wave and the depth of the observed boundary in the body. 6

\footnotetext{
${ }^{5}$ For the sound wave of frequency $1.5 \mathrm{MHz}$ propagating in water the resolution is $2 \mathrm{~mm}$. In children's cardiology for wave of $7 \mathrm{MHz}$ the resolution is $1 \mathrm{~mm}$ and in ophthalmology for the wave of $20 \mathrm{MHz}$ the resolution is $0.2 \mathrm{~mm}$.

${ }^{6}$ To resolve echoes from two surfaces lying in water at depth of $10 \mathrm{~cm}$ in the same plane perpendicular to the direction of sound propagation with the sound wave of $3 \mathrm{MHz}$ the pulse width should be at least $1 \mathrm{~cm}$. The resolution is than $5 \mathrm{~mm}$.
} 


\section{MODE OF SIGNAL PRESENTATION}

The ultrasound diagnostic method is inherently 2D tomographic imaging method. The ultrasound reflected on the consecutive boundaries the detector measures as the voltage on the piezoelectric crystal in the time intervals needed for the sound wave to reach from one to the other boundary. That voltage is than amplified and presented on the monitor as the function of time. There are several possible ways of presenting the results (Fig. 8).

A-mode presents amplitudes of reflected ultrasound in dependence of time. Because the time is direct measure of distances between the detector and the reflection surface, Amode presents the distribution of reflecting boundaries along the direction of propagation.

B-mode presents the echo intensity as the spot of different brightness on the oscilloscope. The Y-axe of the oscilloscope does not react on the signal amplitude, but because the electron bundle is thicker for higher amplitude, the spot is brighter. The distance between the spots reflects the distance between reflecting surfaces. The brightness of the spot is a semiquantitative measure of impendency relationship for the

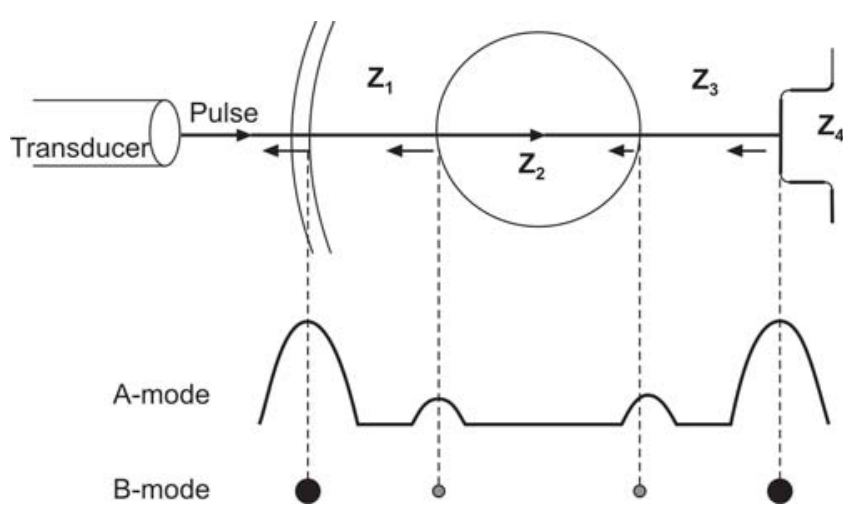

Fig. 8: A- and B-mode in echosonography: The US-pulse propagating through the tissues of different acoustic impedances is partly reflected and partly transmitted at the borders. Echoes are detected in the transducer. On the oscilloscope monitor the incoming echoes are presented in the dependence of the arrival time. The distances between the echoes reflect the distances between the reflecting interfaces. The intensities bear semiquantitative information about the nature of the interfaces. The intensities are presented either by the echo amplitudes, A-mode or by spot brightness, B-mode media building the reflecting interface. The resolution is less. The oscilloscope sensitivity can be adjusted so to presents only the echoes with the amplitude higher than determined threshold. That is bimodal presentation. All spots are the same brightness or not presented. This presentation has a good resolution but is lacking the data of interfaces nature. Higher threshold of intensity eliminates all the reflections on the interfaces with similar impedances and presents only the interfaces of great difference. With lower threshold the data bear information about inhomogeneities inside the tissues. If than the grey scale ${ }^{7}$ is added the image contains information about structure and nature of the interfaces.

In the B-scan mode both directions on the screen, $\mathrm{X}$ and $\mathrm{Y}$, correspond to anatomical distances. Two-dimensional image is constructed from sequential A-scans, obtained over a range of directions, sector scans, or different positions, rectilinear scans. While the A-scan is a single line investigation, B-scan is 2D composite image, and is most commonly used for ultrasound diagnostic imaging.

B-mode images are presented in real time. The probe is either rotated in different angles at one spot at the body surface or it is controlled moved along the body surface (Fig. 9). By combining the translation and rotation the whole body can be scanned. Probe movement is controlled by the electronic devices. For instantaneous measurements of whole sector a probe with more than one piezoelectric crystal is used. The images are produced by successively exciting groups of piezoelectric elements. The image is produced in real time and can even follow the movement inside the tissues: the respiratory, blood flow or movement of diaphragm or the heart.

M-mode or M-scan is combined mode to visualize rapid movement of some reflecting interfaces. The M-scan consists of repeated A-scans, along the same direction perpendicular to moving boundary. The boundary movement changes the echo time detection and that fluctuations are recorded as the displacement in $\mathrm{Y}$ axe in dependence on time, on $\mathrm{X}$ axe. The gained images are not in real time, but provide us with quantitative information about reflecting borders displacement. The exactness of the measuring is not limited to uniform, harmonic movement as by Doppler effect detection. M-mode is used to follow the action of heart, its structures and specially the fetus heart.

\footnotetext{
${ }^{7}$ In grey scale - the different amounts of echoes intensities are presented in shades of grey adding the information about the nature of interface to the image. But the intensities are decreasing because of the audible energy absorption along the propagation path and therefore the intensity is not reliable parameter for diagnostic. To correct for absorption within the body a special amplifier compensates the intensities by increasing the gain logarithmically in time. Some artefact can be result if the attenuation varies significantly, for instance the structures behind the high absorbers will be undercompensated and appear dim. But at the same time this artefact can help to identify more easily some objects, like kidney stones.
} 


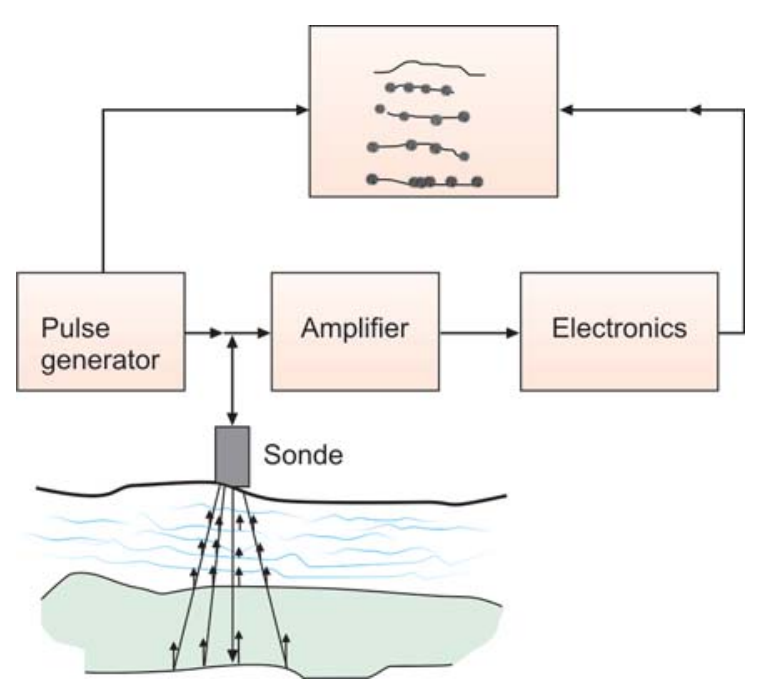

Fig. 9: 2D echosonography: There are more piezoelectric crystals in one probe and each of them is monitoring one of the line in the tissue section. The presentation is in B-mode and the gained image is the real time presentation of perpendicular interface segments. If the probe is rotated or simultaneously translated, the amount of information if multiplied

\section{IMAGING BY DOPPLER EFFECT}

In medical application the Doppler effect is applied mostly for detecting the blood flow. To determine the velocity of the blood the sound waves reflected on the erythrocytes are measured. The erythrocytes scatter the ultrasound in all direction but only the backward wave carries the information of the Doppler shift. Besides, the sound beam usually makes a finite angle with the velocity vector of the blood. It can be taken into account by noting that only the component of erythrocyte motion along the sound beam produces Doppler effect. ${ }^{8}$ Therefore the expression for Doppler shift is:

$$
\Delta f= \pm f_{0} \frac{2 v_{g} \cos \theta}{v}
$$

It is very useful to notice that there will be no Doppler shift measured when the sound beam cross the vessel at right angle. Maximum sensitivity would be for as small angle as possible. In practice the vessels are investigated with $\theta=0-60^{\circ}$ and only smaller segments of vessel are observed. The data are presented as the distribution of the velocities in the cross-section of the vessels (Fig. 10).

There are two technological methods for application of Doppler effect for measuring movements of inner organs. First is named continuous wave (CW) method, because the system

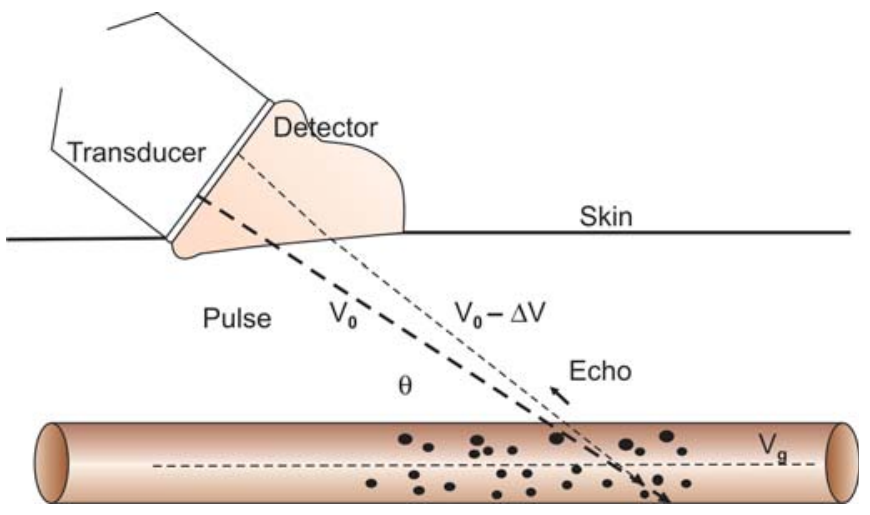

Fig. 10: Doppler shift measurement: The US-wave emitted at frequency $f_{0}$ and speed $v$ is reflected back to the transducer at lower frequency from a boundary moving to the right (away from the source) at the speed $v_{b}$. The change in the frequency is proportional to the speed component of moving erythrocyte in the direction of the ultrasound beam

is sonicated and the echoes are detected continuously. There are separate piezocrystals for emitting and for detecting transducer. The continuous-wave Doppler method cannot distinguish where the echoes come from what is the problem when two vessels are overlaid. But by this method all the different velocities can be detected.

Another method is pulsed Doppler, PW. The sound energy is emitted into the body in short coherent sinusoidal pulses. The reflected signal range gated and demodulated to determine Doppler shift. Gated means the echoes are collected after the time necessary for ultrasound to reach a defined depth in the body. So the exact position of the reflector is determined. The pulse repetition frequency (PRF) is typically in order of few kilohertz. The disadvantage of PW method is that there is an upper limit to the Doppler shift that can be detected. The threshold is determined with sampling rate. All the echoes must be collected before the next pulse is transmitted. The relation of interesting parameters is:

$$
v_{m} z_{m} \leq \frac{v^{2}}{8 f}
$$

where $v_{m}$ and $z_{m}$ are the maximal velocity that can be detected and the maximal depth of penetration and $f$ is the frequency of the sound wave.

Special presentation is a blood flow by the combination of sector B-mode real-time tissue imaging and Doppler frequency shift distribution. The 2D image offers the angular information and vessel size and than the Doppler shift signal quantitatively

${ }^{8}$ Doppler shift is often in audible range. If the ultrasonic frequency is $10 \mathrm{MHz}$, and assuming the blood speed $10 \mathrm{~cm} / \mathrm{s}$, the Doppler effect is $1.33 \mathrm{kHz}$ for the ultrasonic speed in tissue $1500 \mathrm{~m} / \mathrm{s}$. 
interpret the total blood flow and local flow velocity. The presentation is the combination of a grey scale anatomical presentation enriched with the color-coded Doppler signal. ${ }^{9}$ The both information are gained with the same transducer. First the anatomical background is achieved by sector 2D scan and than the single line for Doppler measurements is selected. The both sets of data are processed and the final image is produced.

\section{THREE-DIMENSIONAL IMAGING}

The ultrasound imaging is inherently 2D method. The 2D slices are measured in real time with the probe which has the array of piezoelectric crystals. If than the probe is rotated and translated over the skin surfaces the real time 2D images can be produced. The reasonable further step was to find the method to produce 3D images and present the morphology of natural 3D organs. The easiest way was to follow the experiences of other imaging methods, especially the usage of mathematical algorithm.

A 3D ultrasound data set can be recorded using a conventional 2D scanner. The data are collected by sweeping the probe over the volume of interest and rotating it at the spot. The ultrasound images are then recorded and archived into the computer with their corresponding positions. Constructing a $3 \mathrm{D}$ data set out of all 2D slices requires knowledge of where the slices are relative to one another a how are they placed in the space. This can be achieved by several methods but all need some sort of position sensing device at the probe. The data of 2D echo distribution in three orthogonal planes and corresponding intensities are arranged in three octagonal planes of Cartesian coordinates. Each data corresponds to a voxel element. In the reconstruction process position and the intensity of each echo are good defined. Data are presented in multiplanar representation which uses echo information from the three orthogonal planes through the observed voxel. It is still not the visual 3D image. There is a family of algorithms for visualization of a 3D data set. They use the imaginary light ray to "read" the sound echoes in the volume block. Different algorithm are used which visualize only the hyperechoic or hypoechoic structures. The algorithm taking into the account the lows of reflection on rough boundaries are applied to visualize structure of body surface. That makes the 3D method astonishing especially when the faces of fetus are observed.

Just a step forward is the 4D ultrasound echography. The fourth dimension is the time. The movement in the body can be followed on the real time bases. The most intriguing is the observation of the behavior of fetus; even the opening of eyelid can be seen.

To reach from 2D to 4D ultrasonic diagnostic the development was mainly in the direction of data processing. The detection of data in all methods is based on single beam consecutive echo detection, and collection of echo intensities and echo distribution in direction of beam propagation.

\section{BIOLOGICAL EFFECTS OF ULTRASOUND}

In diagnostic with ultrasound the mechanical energy of sound wave is partially absorbed by the body and therefore can change some of its properties. The possible harmful consequences are dependent on the amount of energy absorbed. Bioeffects can be classified into thermal and nonthermal effects. While sound wave is propagating in the tissue the friction forces are coursing heating. The intensities in diagnostic usage are to low to produce any biological damage because of heating.

By higher wave intensities cavitations can occur. Cavitation describes the building up and behavior of gas bubbles caused by rapid changes of ultrasound pressure in the tissue. The intensities needed for bursting the bubbles and induction of harmful changes is far over the ones used for diagnostic. So, intensity of $100 \mathrm{~mW} / \mathrm{cm}^{2}$, which is used for diagnostic, has been proven over years of observations to be harmful and ultrasonic diagnostic may be considered noninvasive and safe.

\section{REFERENCES}

1. Shung KK. Ultrasound in Shung KK, Smith MB and Tsui BMW. Principles of Medical Imaging, Academic Press, Inc 1992;78160.

2. Guy C, ffytche D. An Introduction to the Principles of Medical Imaging, Imperial College Press London 2005;267-307.

3. Dowsett DJ and Johnston RE. The Physics of Diagnostic Imaging, Chapman and Hall Medical, London 1998;415-66.

4. Fish P. Physics and Instrumentation of Diagnostic Medical Ultrasound, Wiley, Chichester 1996.

5. Hill CR, Bamber JC, ter Haar GR. Physical Principles of Medical Ultrasonic 2004.

6. Preston RC. Output Measurements for Medical Ultrasound, Springer-Verlag, London 2001.

7. Krestel E: Bildgebende Systeme fur die medizinische Diagnostik, Simens, Berlin 1988.

8. Kurjak A: Fleischer AC. Doppler Ultrasound in Gynecology, The parthenon Pub,Group, NY 1998.

9. Breyer B. Physical principles of the Doppler effect and its application in medicine in Color Doppler in Obstetrics, Gynecology and Infertility, Kurjak A, Kupesic S, Art Studio Azinivic-Medison, Zagreb-Seoul 1999.

\footnotetext{
${ }^{9}$ Color coded Doppler is data presentation in which the direction of blood flow is differentiated by the color. The flow approaching the detector is colored red and departing from it in blue. If the shades of the color present the intensities of the flow we talk of power Doppler presentation.
} 